\title{
INFORMATION RETRIEVAL IMPROVEMENT IN E-LEARNING SYSTEMS USING SEMANTIC WEB
}

\author{
Mohamed Hussein Mohamed Hassan \\ Systems and Computer Engineering Dep., Faculty of Engineering(Girls), Al-Azhar University, \\ Cairo, Egypt. \\ E-mail:m.hussein@azhar.edu.eg
}

\begin{abstract}
:
E-Learning and Web-based education and has become a very important branch of new educational technology. In this paper we present information retrieval improvement in e-learning system using semantic web. This paper focuses on design and implementation of adaptive recommendation e-learning system at the sea ports and use e-learning system and semantic web to deliver the learning objects to learners in an interactive, flexible and adaptive manner. The purpose of this paper_is to help trainer in port community to find not just Learning object but also the type of Learning object that they should appreciate from a lot of content and types based on their priority and needs, Our solution consists of a Recommendation System that provides recommendations to the E-learner and advise a learner with most suitable resources and learning objects based on semantic web technology using a hybrid technique of collaborative filtering and content-based filtering using Semantic web, We are also faced with the need to adapt the learning process to the progress of the e-learner, his preferences, as well as existing competencies, to ensure that the system is both effective and efficient, especially considering the multitude of learning objects in existence, we find that adaptive recommendation E-learning systems using semantic web will help the e-learner to find the information and learning object easily and relatively for their queries and interest.
\end{abstract}

KEY WORDS: Semantic web, Extensible Markup Language, Web Ontology Language, Recourse Description Framework, E-Learning.

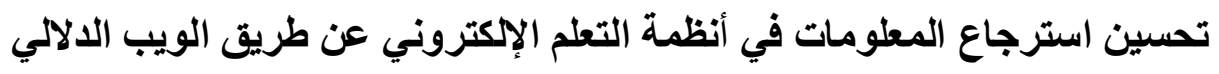

قسم هندسة النظم و الحاسبات ، كلية الهندسة بنات ، جامعة الاز هر ،القاهرة ، مصدر.

البريد الاليكتروني: E-mail:m.hussein@azhar.edu.eg

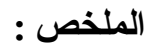

أصبح التعليم الإلكتروني و التعليم القائم على الويب فرعاً هاماً للغاية من تقنيات التعليم الجديدة، واصبح ضرورة ملحة في الوقت

الر اهن. ويركز هذا البحث على تصميم وتنفيذ نظام تعلم إلكتروني يتمتع بخصائص التونية التوصية التكيفية ، بحيث يقوم النظام باختيار

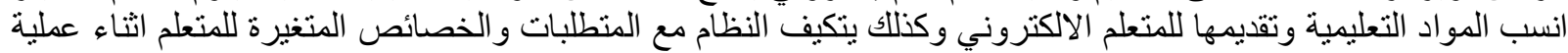

التعلم. وتم تطبيق النظام في المو انئ البحرية وفى ميناء دمياط البحري التئي تحديدا كحالة در اسية وتم استخدام نظام التعلم الإلكتروني

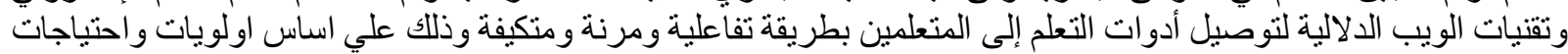

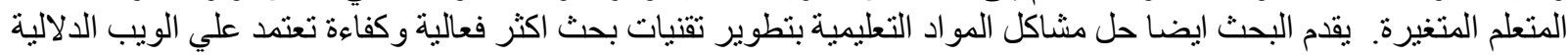




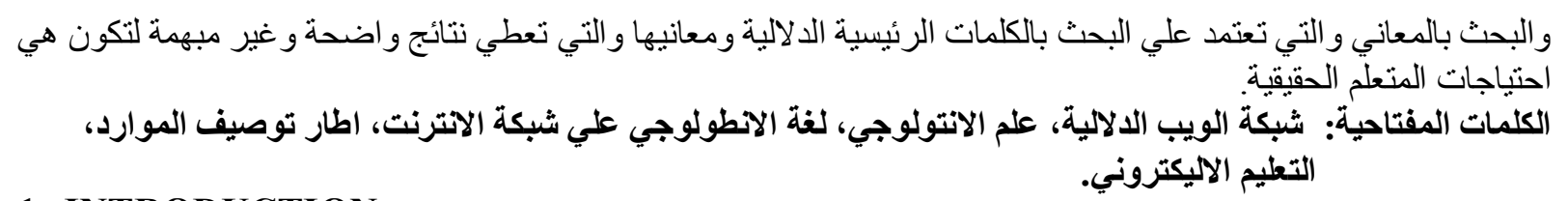

\section{INTRODUCTION}

To offer appropriate learning content to the right learner in a correct way which is a big challenge in Traditional e-learning systems, with deepening informatization of the logistics industry and increasingly fierce market competition, the port enterprises, an important hub for waterway logistics, need to build efficient and flexible information system to gain an edge. In order to increase the operational efficiency and accelerate the flow of goods information, the port enterprises are in need of a complete production management system for the daily handling operations and business activities and a set of information transmission channels for real-time communications with cargo owners, the shippers, customs, and other relevant departments. However, there are several problems in the port informatization at present; A Single Window for trade can be an important facilitation tool. If implemented effectively, it can simplify procedures and formalities for document submission and data collection and can save precious time and money. The training is one of The Most Critical Challenges and Requirements for the Establishment of electronic Single Windows for Handling Export/Import in seaports, Web-based courses and E-learning offer advantages for users and learner. by making access to learning objects and resources just-in time, very fast, and relevance, at any place or time, E-Learning refers to learning that is delivered or enabled via electronic technology, the major problem existed in current E-Learning is. The amount of time spent searching for the right content, absence of personalization and there is an inadequate search technique for searching the web contents. With the absence of semantic, the difficulty of resources sharing and searching, this is mostly caused by the weak-semantic learning resources and the poor sharing mechanism. Nowadays, to offer appropriate learning content to the right learner in a correct way is a big challenge in Traditional e-learning systems, Main problem in e-learning technology is lack of accuracy, time limitation, information overload and cost. There should be mechanism to structure the huge amount of elearning resources and make it readily accessible and reusable in a personalized way.

This access should be assisted for the various types and levels of the e-learning community, whether they belong to professional training, academic programs, lifelong learners or others. World Wide Web known as web 2.0 is used to create many sites such as blogs, wikis, and web applications. In addition to this, web 2.0 is used in many E-learning Systems (ELSs). However, due to the limitations of web 2.0 nowadays, web 3.0 is used in $\mathrm{s}$ Electronic-Learning Management Systems (ELMS). Semantic web is an extension of the current web, in which information is given well-defined meaning, better enabling computers and people to work in cooperation. With the rapid increasing of learning objects (LOs) in a variety of media formats, it becomes quite difficult and complicated task for learners to find suitable learning objects LOs based on their priority and needs. Traditional methods of search engines are not effective during the recovery of search results. That may not be the user's needs. Traditional search engines cannot understand the text of the user and his interests. Because they rely on a set of keywords that are inserted by the users. For syntactic search to get the desired results with different indicators lead to incorrect results, irrelevant or no results to all users when searching [1]. The World Wide Web was not built for machine consuming. It built for human consuming. Although everything on the Web is not Machine-understandable, its machine-readable [2]. We use the Semantic Web to express information in a machine interpretable, precise form, ready for software agents to share, process, and reuse it. as well as to understand what the terms describing the data mean [3]. That would enable web-based applications to interact both on the syntactic and semantic level. semantic web-based educational systems (SWBES) It represents the next generation of web-based educational systems that aim at providing several improvements on the quality of services by the use of semantic web technology. 
We need to represent the knowledge in different contexts on same web page and adapt to different user's requirements and queries. To achieve that, we need to add new technologies that help machines to understand these documents. These technologies are the semantic web [4] [5]. Semantic Web can help search engines deliver better search results. In the traditional Web environment, each Web page only provides information for computers to display the page, not to understand it. the page is just intended for human eyes. Therefore, traditional search engines are forced to do keyword matching only. Without any semantics embedded in the page. the user gets quite a lot of irrelevant results. To solve this problem, we can extend the traditional Web by adding semantic data to it. Recommender systems have been used for e-learning environments to recommend useful materials to users based on their priority and needs. Pre-test and post-test are used to check the knowledge of the user before and after course. Pre-test determine the awareness about the course initially. Post-test determines learning ability of users;

it also compare the difference of learning performance of users. The success of the Semantic Web Based on the easy creation, integration and use of semantic data [6]. The word semantic implies meaning. For the Semantic Web, semantic show that the meaning of data on the Web can be understand and discovered not just by people, but also by machine (computers). Most meaning on the World Wide Web is inferred by users who read the web pages and the labels of hyperlinks, and by other people who write specialized software to work with the data. The phrases "Semantic Web" stands for a vision, in which computers- software - as well as people can find, read, understand, and use data over the World Wide Web to accomplish useful goals for users [7]. The Semantic Web is the abstract representation of data on the World Wide Web, based on the RDF standards and other standards to be defined.

The main problems of the current ELS are the enormous number of the resources,

lack of enabling the information, and time limitation. It is important to

apply a mechanism for ELS in a way that the information and resources can be accessed and

reused. SW is a technology used to improve ELSs, in order to share, reuse the information easily, flexibly, and in a personalized way. Our solution consists of a Recommendation System that provides recommendations to the E-learner and advise a learner with most suitable resources and learning objects., to offer appropriate learning content to the right learner in a correct way which is a big challenge in Traditional e-learning systems, using a hybrid technique of collaborative filtering and content-based filtering using Semantic web, We are also faced with the need to adapt the learning process to the progress of the e-learner, his preferences, as well as existing competencies, to ensure that the system is both effective and efficient, especially considering the multitude of learning objects in existence. Keyword-based model that is based on keywords matching leads to "too many or nothing "and Users often cannot specify appropriate and exact keywords for a valid query. So there is no way the system can learn what the user means and requires by such queries.

\section{METHODOLOGY}

A research approach is defined as the methodology that can be followed during the research. Our research approach mainly follows the Qualitative Analysis Approach proposed by Taylor and Renner [8]. The qualitative analysis approach attempts to put structure to qualitative data. Qualitative data consists of words and observations, not numbers. Qualitative data collected in this thesis is from Journals such as IEEE and ACM, Proceedings, Publications and trusted websites such as the W3C website. After data collection, we follow Taylor and Renner approach which consists of five steps. They are:

1. Understanding of data.

2. Focusing the analysis by questioning what we want to find out.

3. Categorizing information and identify ideas, concepts and terminology.

4. Identifying the relationship between different categories.

5. Explaining what new things we find and learn. 
Also the research methodologies mix in both Descriptive research and Experimental research. Descriptive research on the other hand is a type of research that is mainly concerned with describing the nature or condition and the degree in detail of the present situation. This method use to describe the nature of a situation, as it exists at the time of the study and to explore the cause/s of particular a phenomenon. The aim of descriptive research is to obtain an accurate profile of the people, events or situations. Data Collection is the methods by which data obtained Include surveys, interviews, questionnaires, and case studies. In addition to literature reviews, we organized face-to-face and additional telephone interviews and teleconferences with members of relevant organization's and committees.

\section{LITERATURE REVIEWS \\ 3.1 E-learning}

E-learning systems using semantic web will help the earner and lecturer to find the information and learning object easily and relatively for their queries and interest [9]. Drucker [10] has defined e-Learning as "just-in-time education integrated with high velocity value chains. It is the delivery of individualized, comprehensive, dynamic learning content in real time, aiding the development of communities of knowledge, linking learners and practitioners with experts". ELearning aims at replacing old-fashioned time/place/content/ predetermined learning with a justin time/ at workplace/customized /on-demand process of learning [11] [12]. An e-learning system can be defined as a social and information technological system that supports learning processes [13]. E-learning is also called Web-based learning, online learning, distributed learning, computer-assisted instruction, or Internet-based learning. Historically, there have been two common e-learning modes: distance learning and computer assisted instruction. Computer assisted instruction (also called computer-based learning and computer based training) uses computers to aid in the delivery of stand-alone multimedia packages for learning and teaching. These two modes are subsumed under e-learning as the Internet becomes the integrating technology [14] [15]. Web-based e-learning systems are normally used by a wide variety of learners with different skills, background, preferences, and learning styles. The learning modules need to consist of suitable learning content modules called learning objects (LOs) which are then included in the learning activity structure. The knowledge that the learner has acquired and needs to acquire are then mapped appropriately to the learning activity mechanism that controls the generation of learning content. With the rapid increasing of learning objects (LOs) in a variety of media formats, it becomes quite difficult and complicated task for learners to find suitable LOs based on their needs and preferences. E-learning is extremely beneficial in providing cost effective education irrespective of time and geographical boundaries [16] [17] [18] To support personalization, recommender systems can be used to assist learners in finding the appropriate LOs which will be needed for their learning. In general, courses in LMSs consist of LOs. LO can be defined as a digital and reusable piece of content used to achieve a learning objective. LO can be a text document, an audio file, a video, a picture, or a complete website. Commonly, LMSs are considered as one-size-fits-all systems as they deliver the same kind of course structure and LOs to each learner [19]. However, each learner has different characteristics such as levels of expertise, learning styles, prior knowledge, cognitive abilities and interests, and therefore, onesize-fits-all systems do not support most learners in the e-learning environment, learners find it difficult to select the learning activities that best meet their criteria. Personalization involves the following key stages they include choosing an appropriate learning approach, depending on the learning style and approach for choosing the appropriate content, in turn these contents need to devise the appropriate teaching activities suitable for that learner.

\subsection{Traditional Web restriction}

When you use a web browser to display a web page. It reads a file containing a linear sequence of characters. Most of which is passed unchanged. Hidden in the original sequence. However, there are instructions which tell the browser how to display images, format text, link to other pages, and so on. These instructions are called "tags". This collection of tags and their attributes is called HTML (Hypertext Mark-up Language) [20]. HTML is the standard mark-up language 
used to create web pages, It was designed to create web pages and to display the data on these web pages. But doesn't focus on what the data is or how the data can be stored or transported in an effective and efficient way.

We use semantic web to improve Library and courses Search. The architecture presented in this paper is considered an adaptation model that converts from syntactic search to semantic search. We apply the training at Damietta port in Egypt as a real-world case study. we present one of possible applications of semantic web to management of Web Services, this model semantic webbased educational systems(SWBES)It represents the next generation of web-based

educational systems that aim at providing several

improvements on the quality of services by the use of semantic web technology.

The content of the WWW may be classified into documents and data [21]. Where data may be processed by machines to make them readable and can be handled by the human. And documents are everything readable by the human like reports and mails. Search engines are optimized to retrieve specific per-defined and precise specifications. So that, the end users should know exactly what words to use and the search result for these words will be precise and accurate. It is assumed the end user knows the exact terms to investigate. This suitable in a basic retrieval model, but if the user does not know exactly what words to use in the search engines then the traditional search tools will not useful. Today's World Wide Web (WWW) includes web applications, blogs, wikis, social networking sites, video sharing sites, hosted services it known as Web 2.0. it also includes E-learning Management system and Web-based courses. Research works in the field of web based training and E-Learning are represented by a wide spectrum of applications, ranged from virtual classrooms to remote courses or distance learning. Web-based courses offer advantages for learners by making access to resources and learning objects very fast, just-in-time and relevance, at any time or place. Much progress has been made about the web and related technologies in the past two decades, Web 1.0 as a web of information, web 2.0 as a web of communication, web 3.0 as a web of co-operation and web 4.0 as a web of integration are introduced as four generation of the web since the advent of the web [22]. Because of many of limitations using web 2 for creating web based training and E-learning, we use Web 3.0 which is known as Semantic web. It is a platform to represent E-learning management system that recovers the limitations of Web 2.0.

The Semantic Web may be considered as an evolution to this WWW which aims to make all the information and application data on the internet universally shared and machine process able in a very efficient way. It is an intelligent web which can understand the information semantics and services on the Internet by applying technologies and enabling inference rules to increase users' satisfaction while searching the web content [23] [24]Motivations for inventing the Semantic Web are the limitations of the traditional Web ,Semantic Web provides solutions for these drawbacks by offering more efficient technologies such as XML, RDF and Ontology, The Semantic Web was introduced by Tim Berners- Lee who invented the traditional World Wide Web [25] [26] .

\subsection{Semantic Web Technologies}

The Semantic Web is the extension of the WWW. that allows people to share content beyond the boundaries of websites and applications. There are many different ideas about what the Semantic Web is. Berners-Lee, Hendler and Lassila (2001) define the Semantic Web, also known as Web 3.0, as "not a separate Web but an extension of the current one, in which information is given well-defined meaning, better enabling computers and people to work in cooperation" [27]. Berners-Lee hopes that eventually computers will be able to use the information on the Web, not just present the information. "Machines become capable of analyzing all the data on the Web the content, links, and transactions between people and computers". Based on his idea, the Semantic Web is a vision and is considered to be the next step in Web evolution. It is about having data as well as documents on the Web so that machines can process, transform, assemble, and even act on the data in useful ways. One of the great promises of the Semantic Web is flexibility in 
accessing and identifying information [28]. The Semantic Web is an extension of the current Web. It is constructed by linking current Web pages to a structured data set that indicates the semantics of this linked page. A smart agent, who is able to understand this structure data set, will then be able to conduct intelligent actions and make educated decisions on a global scale.

The Semantic Web takes the solution furthermore. It involves publishing in languages specifically designed for data: Resource Description Framework (RDF), Web Ontology Language (OWL), and Extensible Markup Language (XML). In contrast with the simple HTML, the XML allows content creators to label information in a meaningful way. but the machine still knows nothing about what is meant by this structure. The RDF then comes to represent the relationships between the data items and give more meaning for the XML labels. The OWL provides the semantics for the data syntax and solves some problems occurred such as "two databases may use different identifiers for the same concept". Then the machine needs OWL to discover the data which have the same meaning. These expressive technologies enable the data to be represented in a machine readable structure and hence enable the machine applying inference rules on the data to obtain meaningful results to improve the search process. Briefly, the Semantic Web is supposed to make data located anywhere on the Web understandable and accessible, both by people and machines.

\subsection{THE SEMANTIC WEB ARCHITECTURE}

Web based Learning Management Systems should focus on how to satisfy the e-learners needs. And it may advise a learner with most suitable resources and learning objects. Nowadays, to offer appropriate learning content to the right learner in a correct way is a big challenge in Traditional e-learning systems But Because of many limitations using web 2.0 for creating E-learning management system, nowadays we use Web 3.0 that is known as SW (Semantic web). selecting appropriate learning services for a learner from a large number of heterogeneous knowledge sources is a complex and challenging task, The term "Semantic Web" involve efforts to build a new World Wide Web architecture that enhances content with formal semantics, means the content is made suitable for machine only, as opposed to content that is only intended for human. This will enable agents to cause about the Web content, and make an intelligent response to unexpected condition. the main task of the Semantic Web is "Expressing meaning", In order to achieve that objective several layers of representational structures are needed, they are presented in the Figure 1, among which the following layers are the basic ones [26]. The World Wide Web architecture can be divided into 3 parts which are [29] [30].

1. Knowledge representation (XML, XML schema and RDF, RDF schema layers).

2. Ontology (Ontology vocabulary layer).

3. Agents (Logic, Proof, Trust layers).

The Semantic Web can be exploited as a very suitable platform for implementing an E-learning system, because it provides all means for E-learning: ontology development, ontology-based annotation of learning materials, their composition in learning courses and active delivery of the learning materials through E-learning portals [31].

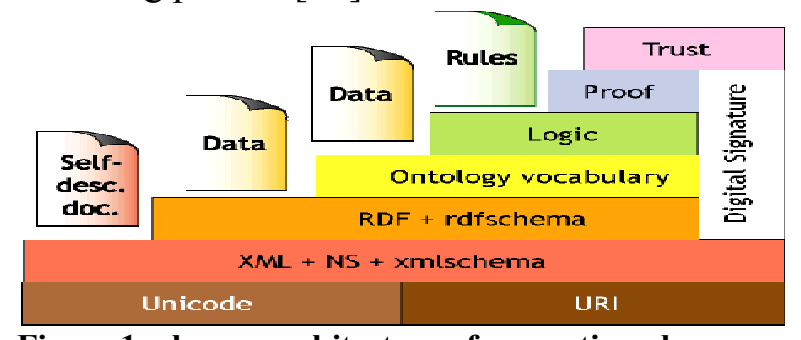

Figure 1 - layers architecture of semantic web

Figure1 shows the nine layers' architecture of semantic web in which the lowest layer starts from the bottom of the layer, followed by the highest layer, which is at the topmost position. The various layers and its challenges are described below. 


\subsubsection{UNICODE}

Unicode is the basic universal number for every character, which works in multiple platforms. It is the basic notation, which is supported by top multinational companies like Motorola, IBM, Intel,etc. Unicode allows a single software, text or single character to be transported to other parts without corruption and re-engineering.

\subsubsection{URI (Uniform Resource Identifier)}

The URI is termed as Uniform Resource Identifier, which is a basic syntax for strings that is used to identify a resource. A resource is any physical or abstract things in which each item has an identifier. The URI consists of two types: First is Uniform Resource locators (URL) which identifies a resource and how it can be accessed, and the second part is Uniform Resource Names (URN)that is used to create a universal and persistent name about a resource in its namespace. This namespace dictates the syntax of URN identifier [31]. It is used to identify resources on the web, in which every resource in the WWW should be uniquely identified so we give it a URI. Resources could be anything such as a document, book, or video. There are different forms of the URIs. The most familiar form is the URL which is typed in the Web browser to locate its corresponding resource, so it has two functionalities which are identifying and locating the resources. There are other forms that only identify the resources but can't tell us their locations. Because the Web is too large to be controlled by only one person or organization [32], so creation of URIs is decentralized and anyone could create URI for her resources, It is clear that a problem of identifying the same resource with more than one URI may exist, but it is the cost of having such flexible and simple technique to identify resources on the Web [33].

\subsubsection{XML (Extensible Markup Language)}

XML is aW3C-recommended general-purpose markup language that supports a wide variety of applications, XML is also designed to be reasonably human-legible, and to this end, terseness was not considered essential in its structure. XML is evolved from simplified subset of Standard Generalized Markup Language (SGML). XML facilitate the sharing of data across different information systems, particularly systems connected via the Internet. XML is the simplest way to send the document across the web to its specific format. It allows users to edit or modify it and again transfer it. These document formats can include markup also to enhance the meaning of the document [34] XML is the primary and core foundation of the semantic web will be built on the top of XML. Scientifically, XML is built upon Unicode characters and URI's. The Unicode characters allow XML to be characterized using International characters [35].

\subsubsection{XML Schema}

XML Schema is a document definition language that enables you to develop XML documents into a specific hierarchical structure and vocabulary, the things you want to define in your language are element types, attribute types, and the composition of both into composite types (called complex types). XML Schema is different to database schema, which defines the column names and data types in database tables. XML Schema has been approved by W3C consortium in the year2001, XMLS allows the validation of instances to ensure the accuracy of field values and document structure at the time of creation, The accuracy of fields is checked against the type of the field; for example, a quantity typed as an integer or money typed as a decimal, The structure of a document is checked for things like legal element and attributes names, correct number of children, and required attributes, All XML documents should be checked for validity before they are transferred to another partner or system. There are different types of XML Schema Languages.

\subsubsection{XML Namespaces}

An XML namespace is the $\mathrm{W} 3 \mathrm{C}$ recommendation for providing uniquely named elements and all of its attributes in an XML instance. An instance of an XML contains element or attribute names from more than one vocabulary. If each vocabulary is given a namespace, then the uncertainty or what is unexpected between identically named elements or attributes can be resolved. All the elements which are within a namespace must be in unique component [36]. 


\subsubsection{RDF and RDF Schema}

Resource Description Framework (RDF) is a foundation of metadata processing. It provides interoperability between applications that exchange machine-understandable information on the Web [37] [38], and it defines the relationship between the resources on the web. RDF can represent by different syntaxes, one of the most popular syntax is the XML where the RDF based on this syntax is called RDF/XML model, The RDF statement is written in a triple form consisting of three parts which are the subject, the predicate and the object, so it seems like it is a natural phrase but its parts are URIs as they are resources on the Web. A syntax (which is XML) representing this model is required to store instances of this model into machine-readable files and to communicate these instances among applications RDF imposes formal structure on XML to support the consistent representation of semantics. The RDF and RDF Schema layer is located above the XML layer, which provides more functions and capabilities than in XML and XML schema. The Resource Description Framework is an XML based language that is used to describe resources. Such a resource is identified via a uniform resource locator (URL). As compared to XML documents that attach the metadata of the document, RDF captures the metadata of the externals of the document such as author, creation, date, etc. [31].RDF is the machine process able language unlike XML which is human process able and it is not understandable by humans i.e. it knows what the machine is doing in the way it does. So that it can store smart information back on the Web. [39], Resource Description Framework Schema (RDFS) is a knowledge representation language, submitting basic elements for the description of ontologies [40].

\subsubsection{Ontology Vocabulary}

The term "ontology" can be defined as an explicit specification of conceptualization [41] [42], the conceptualization means modeling certain domain and the Ontology is used to describe important concepts of this domain, so it is the specification of this conceptualization. Ontology is the stage where the vocabularies related to a specific domain should be defined. It provides the capability to make analysis on the relationships between the vocabularies to discover problems such as the existence of two vocabularies of the same meaning. In this stage the relationships between vocabularies of a specific domain are created in hierarchal form by using the inheritance and classes concepts. Languages such as OWL (web ontology language) which may be considered as a syntactic extension for RDF/RDFS are provided at this stage. The main layer of semantic web architecture is Ontology vocabulary, which typically consists of hierarchical distribution of important concepts in a domain, along with descriptions of the properties of each concept. OWL (Web Ontology Language): OWL is intended to be used when the information contained in documents needs to be processed by applications, as opposed to situations where the content only needs to be presented to humans [43]. OWL can be used to explicitly represent the meaning of terms in vocabularies and the relationships between those terms. This representation of terms and their interrelationships is called ontology. OWL has more facilities for expressing meaning and semantics than XML, RDF, and RDF-S, and thus OWL goes beyond these languages in its ability to represent machine interpretable content on the Web, OWL has been designed to meet the requirements of RDF, RDFS, XML Schema [44].

\subsubsection{Logic Layer}

Logic layer in the semantic web is the universal language of monotonic logic. In the logic layer of semantic web architecture any rule can export the code but cannot import it. Any system in the logic layer can validate proofs [45]. This layer functions on the basic principle of first order predicate logic, so the information is displayed accurately on the web.

\subsubsection{Proof}

In this layer, the final goal of semantic web is to create smarter content which could be understood by the machines. When the machine understood the content, some confirmation may come out of the content and new information will be produced.

\subsubsection{Trust}

The trust layer in semantic web architecture is analyzed into four parts: The Information integration layer handles aggregation of information from different sources and adds provenance metadata in the information. The Repository Layer stores the aggregate information. 


\subsubsection{Digital Signature}

Digital signatures layer run horizontal to the RDF family up through the layer proof and support the notion of trust. The main purpose of the digital signature is to digitally sign the document. Also encryption methods are used to run on digital signed documents to prevent against unauthorized access.

\section{CASE STUDY : E-LEARNING BASED ON SEMANTIC WEB TECHNOLOGY}

The Semantic Web using as a backbone for eLearning. Foremost, the objectives are to ease the contribution of and the efficient access to information. But, in general, a Semantic Web-based learning process could be a personalized (user customized), relevant (problem-dependent), and an active (context-sensitive [46]. We will describe the general model of solution in this section in details to understand how smart Search model retrieve the learning object.

\subsection{Library Search: the proposed Model}

The Library Search model is an example of semantic search engine based on semantic agent. The proposed model will effectively help users to find correct LO based on the semantic query. Which are implicitly and seamlessly injected into both user query and Library Search concepts stored in RDF. in contrast with traditional query which depends on keywords only, and not their meanings. The Library Search model is composed of two tiers, with each tier is responsible for specific task. The tier itself may be composed of multiple components. The Two tiers are structured as follows:

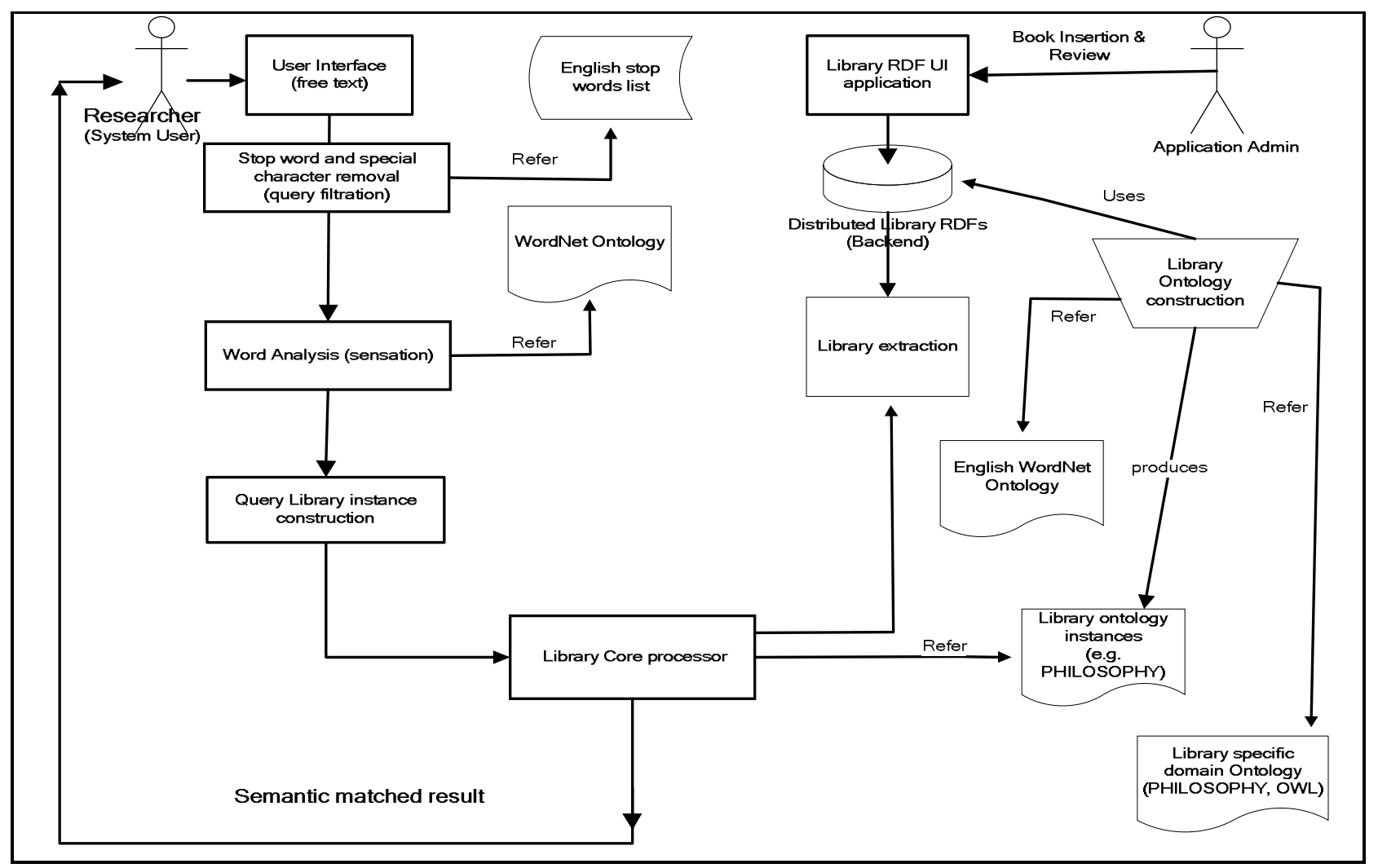

Fig 2: proposed system Architecture details

\subsection{The presentation tier}

This tier is the top most level of the application. The presentation tier displays information related to Library search query and answers. This tier contact with other tiers by outputting results to the browser/client tier and all other tiers in the network. The Presentation layer has been implemented using J2EE technologies such as GWT, RPC and CSS. 


\subsection{The business logic tier}

This tier controls the application's functionality by performing detailed processing, handling transformation of input text, information exchange between the RDF data, the knowledge base (ontology) and the user interface. It Consisting of A group of agents that interact together to achieve a specific complete task related to a specific Library Search domain. An agent is a software component that has autonomy that provides an interoperable interface to other agents in the system, besides, it is adaptive, versatile and performs some diverse tasks (such as matching, ranking, parsing Ontology, reasoning over data etc...). This tier is decomposed into four main components described in the following subsections.

\subsection{The stop words removal component (query filtration)}

When the user enters his query in the text area, he may enter other special characters such as punctuation marks. This component is responsible for filtering all special character and punctuation marks. The query words are separated by Stop words such as: if, with, on, from or the. Stop words are words which are filtered out prior to query processing. These words are eliminated by referring to a list contains stop words. Example of a query (Dynamics and Steady States in excitable mobile agent systems). After removing the stop words. The query becomes (Dynamics Steady States excitable mobile agent systems). The cause for eliminating the stop words is that these words do not add any crucial information and are not indexed.

\subsection{The word analysis component (sensation)}

This component is the first semantic component that lists the words in the query. And fetches their different meanings or references, by referring to predefined Word Net ontology for Library book terms and axioms used in any Library search domain. We mean by the term "different meaning", that a word may be lexically equivalent and consists of the same character, but with different meaning. As an example, the word (Car). This has meanings (Truck) and (Land carrier). This component works as follow, first it tries to disambiguate word sense by using one of classification algorithms. if single definition is found, it processes this definition; otherwise it pops up a window proposing the word meaning to the user, with the facility to let the user select another meaning. Different WSD algorithms can be plugged here. Knowledge retrieval: input query is annotated according to user profile and interest then by using ontology, semantically relevant information is retrieved, Semantic web is used to make the-learning system more responsive and interactive.

\subsection{The Query Library instance construction component}

As the query is normalized, another component begins the work of constructing anonymous domain instance that represents this query. in order to be matched against stored books names instance in knowledge base (RDFs). This component is responsible for this task.

\subsection{The Library core processor component}

This is the most important component in the model. It is the cornerstone of the system which performs multiple operations. including Library documents preparation, RDF indexation, instance ontology matching, Library ontology instance references, preparing the matched result to be sent to end user. Performing some calculations to calculate the ratio of semantic distance between the question and the returned results and finally sorts the result according to their interested data field.

Personnel knowledge search engine personalize knowledge according to learner profile and support to search learning path suitable for user. Knowledge service functionality has been integrated with semantic web service. Ontology discuss here is developed in the Protégé tool. It is freely available, open source, having a great set of plug-ins, GUI based and also popular. The aim of this research is to improve the learning experience and learning outcomes via Design and Implementation of knowledge Base Industrial Adaptive Recommender E-Learning System Using Semantic Web. The important contributions of this paper are:

1. We have designed and implemented a successful information retrieval and e-learning system based on semantic web technology in Damietta port at Egypt.

2. Improve information retrieval and e-learning system using semantic web technology. 
3. A user has access to the training and suitable learning objects from anywhere in the world using the Internet, breaking the limits of the number of participants on site and distance.

4. Using semantic web to help user to Minimal service execution time, Guaranteed, permanent connection with service, and Guaranty of confidentiality and secure private information exchange.

\section{CONCLUSION}

The proposed system to solve the limitation of current e-learning environment by providing most relevant learning resources to e-learner. In order to do this, the user's profile is compared to some reference characteristics. These characteristics may originate from the information item (the content-based approach) and the user's social environment (the collaborative filtering approach). In this paper we represent a part of our research work, and proposes an adaptive recommendation system for information retrieval based on different methods and techniques from the following communities to achieve the model: e-learning, ontology, Semantic Web and Web Services, Mobility, and Word Net, adaptive learning, and recommendation system; it is applied on elearning and Library search domain. In this paper we focus on design and implementation of knowledge-based industrial reusable, interactive, web-based training activities at the port and logistics sector and use e-learning system and semantic web to deliver the learning objects to learners in an interactive, adaptive and flexible manner. The system presented in this paper is considered an adaptation model that converts from syntactic search to semantic search. We have applied the training at Damietta port in Egypt as a real-world case study. The system advises a learner with most suitable learning objects. We use semantic web to improve e-learning and courses Search by using adaptive recommendation system. The main purpose of our paper was to illustrate possibilities of using the Semantic Web as a backbone for E-Learning. And provides flexible and personalized adaptive access to the learning materials. The main contribution is our model for Improve information retrieval and e-learning system using semantic web technology. The Used Technologies are presented in this paper significantly contribute to the limited literature on adaptive recommendation e-learning using semantic web. We implement the system using the new technologies in Semantic Web and evaluate the performance against traditional E-learning systems.

\section{Future work}

In the future, we will improve the system by using recommendation and improve keyword clustering algorithm through identifying synonyms among keywords we may will make some improvements to the weighted keyword algorithm based on interest profiling approach and the subject ontology extension method and use adaptive recommendation learning.

\section{REFERENCES}

1. D. F, H. Ian, and V. Frank, M. Deborah and F. Peter, 2001, "Ontology Infrastructure to Enable the Semantic Web," IEEE Intelligent Systems, vol. 16, no. 2.

2. O. Lassila, 1998, "Web Metadata: A Matter of Semantics.", IEEE Internet Computing, vol. 2, no. 4, pp. 30-37.

3. S. R. Linta, M. M. Islam and M. R. Islam, 2012 "An Enhanced Model of ELearning Management System Using Semantic Web Technology and Development of Universal Namespace for University Domain," IJCSI International Journal of Computer Science Issues, vol. 9, no. 2.

4. J. Heflin, 2001, "Toward the Semantic Web: Knowledge Representation in A dynamic, Distributed Environment," Ph.D. Thesis, University of Maryland, College Park.

5. S. Decker, S. Melnik, F. Harmelen and D. Fensel, 2000, "The Semantic Web: The Roles of XML and RDF," IEEE internet computing, vol. 4, no. 5, pp. 63-75.

6. T. David and . W. Johanna, 2006, "Web semantics and ontology ISBN: 1-59140905-5," Idea Group. 
7. Thomas B, 2004, "Explorer's Guide to the Semantic Web," Manning, ISBN 1932394-20-6.

8. E. Taylor-Powell and R. Marcus, 2003 , Analyzing Qualitative Data. Program Development and Evaluation, Madison, Wisconsin: University of Wisconsin, Extension.

9. G. Shrivastav, K. Sharma and A. Bawankan, 2012 "A new framework semantic web technology based e-learning," in In Environment and Electrical Engineerin(EEEIC).

10. P. Drucker, 2000 "Need to Know Integrating e-Learning with High Velocity Value Chains," A Delphi Group White.

11. L. Stojanovic, S. Steffen and S. Rudi, 2001, "eLearning based on the Semantic Web," in WebNet'2001 World Conference of the WWW and Internet.

12. N. Kontodimopoulos, A. BOUKOUVALAS1, K. Savidakis and A. Gasparinatou, 2004, "Distance-Learning Educational Material in the Biomedical Engineering Degree Program," in WSEAS Int. Conf. on ENGINEERING EDUCATION, Venice, Italy.

13. Hoppe, G.; Breitner, M. H, 2004, "Business Models for E-Learning," in Elearning,models, istrument,experiences, of the Multikonferenz Wirtschaftsinformatik, Essen-Germany.

14. G. Jorge, M. Ruiz and J. Michael, 2006, "The Impact of E-Learning in Medical Education," in Academic Medicine.

15. Agelesslearner, 2015, "e-Learning- Introductions,". [Online]. Available: http://agelesslearner.com/intros/elearning.html.

16. M.-L. Hung and ,. C. Chou, 2015,"Students' perceptions of instructors' roles in blended and online learning environments: A comparative study," Comput. Educ, vol. 81, pp. 315-325.

17. Y. M. Cheng, 2011, ",Antecedents and consequences of e-learning acceptance," Inf. Syst. J, vol. 21, pp. 269-299.

18. J. L. Chen, 2011, "The effects of education compatibility and technological expectancy on e-learning acceptance," Comput. Educ, vol. 57, no. 2, pp. 1501-1511.

19. S. Shishehchi, S. Banihashem and N. Zin, 2011, "Review of personalized recommendation techniques for learne in e-learning systems," in Proceedings Proceedings of the International Conference on Semantic Technology and Information Retrieval (STAIR, Putrajaya).

20. U. Magnusson, 2013, HTML in 30 Pages.

21. "Semantic_Web,"2013,[Online]Available: http://en.wikipedia.org/wiki/Semantic_Web.

22. S. Aghaei, M. A. Nematbakhsh and H. K. Farsani, 2012, "Evolution of the World Wide Web : from web 1.0to web 4.0," International Journal of Web \& Semantic Technology (IJWesT), vol. 1, p. 3, anuary.

23. P. P. I. Horrocks, 2003, "Three Theses of Representation in the Semantic Web,"," in Proceedings of the 12th international conference on World Wide Web.

24. L. Yu., 2007, Introduction to the Semantic Web and Semantic Web Services, Taylor \& Francis Group, LLC.

25. G. Ross, [Accessd 14 2013], "An introduction to Tim Berners-Lee's Semantic," 31 January 2005. [Online]. Available: http://www.techrepublic.com/article/an-introductionto-tim-berners-lees semantic-web/5552998. 
26. T. Berners-Lee, 1988, "Semantic Web Road map,". [Online]. Available: http://www.w3.org/DesignIssues/Semantic.html..

27. T. Berners-Lee, J. Hendler and O. Lassila, 2001, "The Semantic Web: A new form of Web content that ismeaningful to computers will unleash a revolution of new possibilities.," Scientific American.

28. B. Ö. Czerkawski,4, October 2014, "The Semantic Web in Teacher Education", TOJET: The Turkish Online Journal of Educational Technology, vol. 13.

29. M.-B. T, 2004, Everything Integrated: A Framework for Associative Writing in the Web, University of Southampton.

30. A. B. A. J. v. d. M. A. Gerber, 2008, "Functional Semantic Web Architecture,"," Springer Berlin / Heidelberg, Lecture Notes in Computer Science, Vols. Volume 5021/2008, ISBN:978-3-540-68233-2, pp.: 273-287.

31. L. O. K. T. S. Michael Daconta, 2002, "The Semantic web: the guide to future of XML,web services and knowledge management".

32. A. Swartz, 2002, "The Semantic Web in Breadth,". [Online]. Available: http://logicerror.com/semanticWeb-long..

33. R. Pandey and S. Dwivedi, November 2010, "Interoperability between Semantic Web Layers: A Communicating Agent Approach," International Journal of Computer Applications, vol. 12, no. 3, pp. 0975 - 8887.

34. "XML schema,2006"[Online].Available: http://en.wikipedia.org/wiki/XML_schema.

35. T. Bray, 2012, "Extensible Markup Language (XML) 1.1 (Second Edition)," [Online]. Available: http://www.w3.org/TR/xmll1/\#sec-origin-goals.

36. "REC-xml-names, 2013, " [Online]. Available: http://www.w3.org/TR/REC-xmlnames.

37. D. Beckett, 2004, "RDF/XML Syntax Specification (Revised)". [Online]. Available: http://www.w3.org/TR/REC-rdf-syntax/.

38. E. Miller, 1998, "An Introduction to the Resource Description Framework," D-Lib Magazine.

39. [ "Resource Description Framework," [Online]. Available: http://en.wikipedia.org/wiki/Resource_Description_Framework.

40. "RDF-schema," [Online]. Available: http://www.w3.org/TR/rdf-schema.

41. M. Obitko., 2007, "Introduction to Ontologies and Semantic Web,". [Online]. Available: http://www.obitko.com/tutorials/ontologies-semantic-web/.

42. S. S. A. Maedche, 27-9-2001, "Ontology Learning for the Semantic Web," IEEE Intelligent Systems, vol. 16, no. ISSN:1541-1672.

43. W3C, [Accessed 276 2015], "OWL Web Ontology Language Overview,". [Online]. Available: http://www.w3.org/TR/owl-features/.

44. "OWL-features," [Online]. Available: http://www.w3.org/TR/owl-features.

45. "Logic layer," [Online]. Available: http://www.w3.org/2002/Talks/04-sweb/slide200.html.

46. L. Stojanovic,S. Staab and R. Studer, 2001 "eLearning based on the Semantic Web," in WebNet'2001 World Conference of the WWW and Internet. 